(C) 2018 IEEE. Personal use of this material is permitted. Permission from IEEE must be obtained for all other uses, in any current or future media, including reprinting/republishing this material for advertising or promotional purposes,creating new collective works, for resale or redistribution to servers or lists, or reuse of any copyrighted component of this work in other works. DOI 10.1109/VTCSpring.2018.8417755

\title{
Vehicular Networking in the Recursive InterNetwork Architecture
}

\author{
Torsten Braun ${ }^{1}$, Davide Careglio ${ }^{2}$, Ibrahim Matta ${ }^{3}$
}

\begin{abstract}
Vehicles such as cars are expected to use communication technologies for retrieving different kinds of information and exchanging information with other vehicles for safety and infotainment purposes. This results in vehicular networks, where vehicles can connect to other vehicles or communication infrastructures such as Road Side Units. The Recursive InterNetwork Architecture (RINA) has been proposed as a Future Internet architecture. This paper investigates and analyses how vehicular networks can be supported by RINA and how a RINA based vehicular network architecture can be designed to support efficient management of mobile vehicles.
\end{abstract}

\section{INTRODUCTION}

Vehicle networking is intended to enable vehicles to send and receive different kinds of information to support safety (e.g., warnings about possible accidents) but also to provide information about traffic situations such as traffic jams or road conditions. Moreover, information must be provided to passengers, e.g., for entertainment (e.g., videos) or information (e.g., touristic information). Vehicle-to-infrastructure communication will be supported by Road Side Units (RSUs) using a variety of technologies, e.g. WiFi, WAVE/DSRC, $4 \mathrm{G} / 5 \mathrm{G}$, etc.. Alternatives for direct RSU connectivity are multi-hop networks and delay/disruption-tolerant networks (DTN). DTN issues are beyond the scope of this paper. Direct vehicle-to-vehicle (V2V) communication must be supported as well, to enable low-delay messages between vehicles, in particular for emergency indications. The target scenario is depicted in Fig. 1, where vehicles are directly or indirectly (via other vehicles) connected to RSUs.

The Recursive InterNetwork Architecture (RINA) [1], [2] has been designed as a Future Internet architecture. RINA is based on Distributed IPC (Inter-Process Communication) Facilities (DIFs), each consisting of a set of communicating processes [1]. Networking is defined as "Inter-Process Communication" [1] and application processes should be supported by an IPC Facility. RINA has several characteristics and features required for vehicular networks such as efficient multi-homing support [3] and strong security [4]. This paper analyses how vehicular networks can benefit from RINA and how RINA can support vehicular networks.

Sec. II describes application scenarios and requirements. Sec. III introduces the RINA-based architecture for vehicular application scenarios (V-RINA) and discusses the design of V-RINA. Sec. IV analyses the signaling overhead to handle mobility. Sec. V concludes the paper.

\footnotetext{
${ }^{1} \mathrm{~T}$. Braun is with University of Bern, Switzerland braundinf. unibe. ch

${ }^{2}$ D. Careglio is with Universitat Politecnica de Catalunya, Spain caregliodac.upc.edu

${ }^{3}$ I. Matta is with Boston Univeristy, USA matta@bu . edu
}

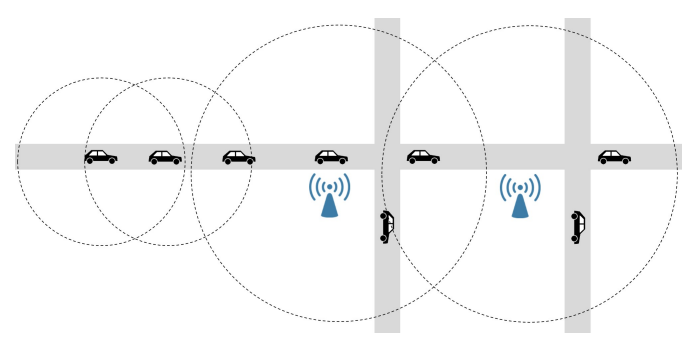

Fig. 1. Vehicular Networking Scenario with Road Side Units

\section{Vehicular Networking Applications}

The basis for many driving decisions and warnings in vehicular networks is the data collected from vehicles and their environment, possibly using sensors. Vehicles should report their location, speed, and directions to allow further processing and traffic analysis. The vehicle should report such data to any, possibly the closest, service reachable via the connected RSU. Such data have to be processed by services running on physical or virtual servers, if possible close to the vehicles as suggested by Mobile Edge Computing [5] to reduce delays. Unicast and anycast communication between vehicles and the collecting server is needed. After data from vehicles have been collected, it can be processed, analysed, and stored at (distributed) services [6]) . Users in vehicles might then be interested to retrieve information about the traffic situation for navigation and adapting travel routes. Moreover, users such as passengers might want to retrieve information for entertainment or additional travel information, e.g., travel guides or videos. In [7] the challenges of content distribution in VANETs are discussed. Infotainment applications can be provided by firstly searching for the desired content and after it has been found by retrieving it from the identified server. Appropriate mechanisms to identify and locate such information as well as services are needed.

The distribution of warnings about events such as hazards, traffic jams, or extreme road conditions within an affected geographic area, uses such data collected from many different vehicles. Such data must be collected from many vehicles to obtain reliable data and to avoid that such information depends on single, possibly malicious, vehicles. Warnings might not only require high bandwidth, but delays should be low in the (milli)seconds range and one has to ensure that all vehicles within a certain area receive such messages. Warning messages might be distributed by an RSU in order to reach all vehicles around it, e.g., for announcing traffic jams. Alternatively, a single vehicle might 
announce a warning, e.g., about fast brake operations, in its direct neighborhood. Geocasting [8], [9], [10] can support efficient message dissemination in geographic areas. Geocast functionality can be implemented in RINA on application level, i.e. within DAFs, possibly using schemes similar is in overlay networks, but it is beyond the scope of this paper.

\section{Vehicular RECURSIVE INTERNETWORK ARCHITECTURE (V-RINA)}

This Section presents a design for a RINA-based vehicular network architecture to support applications discussed in Sec. II. RINA's support for multi-homing [3] and security [4] are important prerequisites for vehicular networks.

\section{A. Example Scenario}

Fig. 2 shows a sample network graph with RSUs distributed over four areas. Two areas form a region. Two regions are interconnected via Region Routers (RR) and a Backbone Router. RSUs may be interconnected in different ways. Area 1 shows a star network, while in area 4, RSUs might form a mesh network. RSUs form a cell and vehicles might directly (e.g., in area 4) or indirectly (e.g., in area 1) connect to a RSU. Two vehicles in area 1 form a simple VANET. Vehicles have to register at the responsible address management entities if they are moving to a new cell, area, or region, respectively. For simplicity, we assume that RSUs, Area and Region Routers are responsible for this.

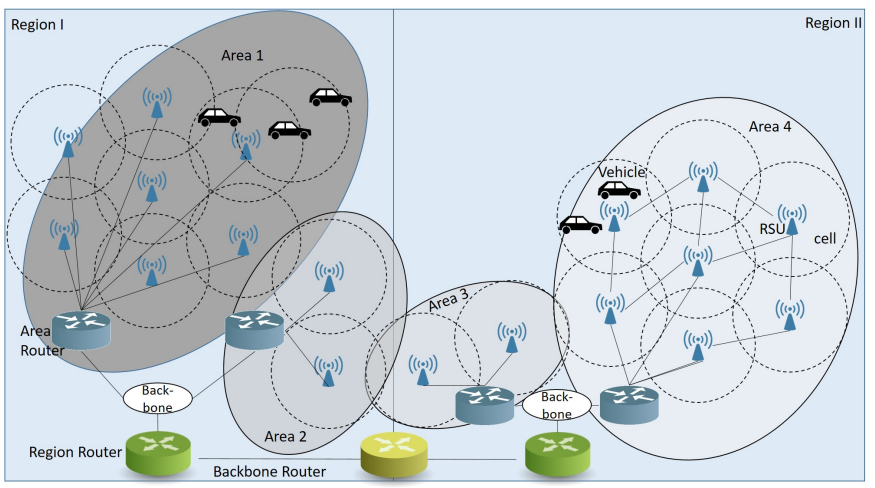

Fig. 2. Example Vehicular Network Topology

\section{B. Distributed IPC Facilities Structure}

Fig. 3 shows the Vehicular RINA (V-RINA) DIF architecture for the network depicted in Fig. 2. On the lower layers we see point-to-point (P2P) links interconnecting routers or vehicles within a VANET. A RSU forms a Cell DIF, which includes all processes at vehicles connected to the RSU as well as those included in the vehicular ad-hoc network (VANET). Vehicles have to register and authenticate when changing RSUs / cells. An Area DIF, which can be managed by an Area Router (AR) or another (distributed) management system, includes all vehicles within the area and all RSUs covering the area. A Region DIF managed by the Region Router (RR) includes all vehicles within that region as well as all ARs. On top of the Region DIF, a Global Network
DIF interconnects all Region Routers (RRs) and all vehicles. For DIF management, we assume a single manager (RSU, AR, RR) in Cell, Area, and Region DIFs, respectively, for simplicity of explanation. Management tasks include address management among others. Such central management scheme can be distributed easily as discussed in Sec. III-C. On the top layer, two Distributed Application Facilities (DAFs) are depicted, one per distributed application. DIFs below the DAFs provide basic network services, primarily routing.

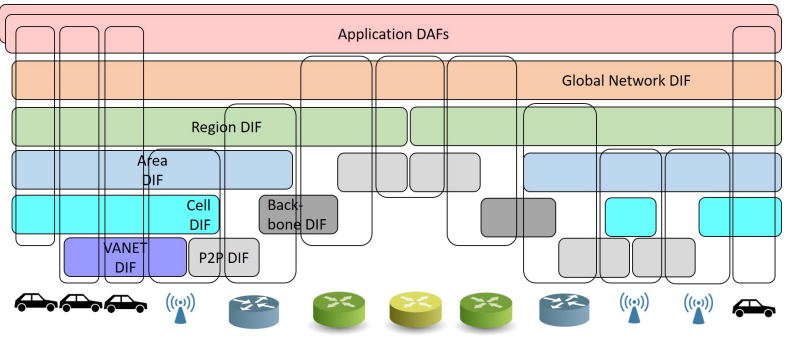

Fig. 3. V-RINA

\section{Naming and Addressing}

At each vehicle there are processes with unique names in the DAF as well as globally unique addresses in the Global Network DIF. Below the Global Network DIF, we introduce a hierarchy of geographical areas to support efficient address management in case of mobility. This hierarchical approach has been motivated by HMIPv6 [11], which has been standardized in RFC 5380. HMIPv6 introduces a twolayer registration hierarchy for Mobile IPv6, but it could be extended to multiple layers. A mobile node registers a Regional Care-of-Address (CoA) at its home agent and correspondent hosts. The Regional CoA is then mapped to a Physical CoA (PCoA) by a Mobility Anchor Point. In case of local mobility, a mobile node only has to update its PCoA. The signaling load, however, depends on the number of correspondent hosts.

We assume that a larger area can be divided into smaller sub-areas, and those can be divided into sub-sub-areas etc. This results in a hierarchy of areas. In the following we assume three hierarchy levels, namely Region, Area, and Cell, for which separate DIFs are established. When a vehicle joins a Region, Area, or Cell DIF for the first time, it acquires an address on each of these levels. This has the advantage that it allows efficient management of mobile vehicles moving between those Regions, Areas, and Cells. Address management overhead of vehicles depends on the DIF layer. The higher the DIF layer the more vehicles must be managed. The frequency of registration changes becomes lower for higher layer (and larger, in terms of geographical size) DIFs, but higher for lower layer (and smaller, in terms of geographical size) DIFs, whereas the number of vehicles per DIF decreases. For larger regions, e.g., a state, a huge number, e.g., thousands or millions of vehicles might be registered, but changes are rather infrequent. On the other hand, for cells, e.g., a block in a city, there might be only a few, e.g., dozens of cars, but changes are rather frequent. 
For addressing on the lower layers (Region, Area, Cell) we suggest topological addresses: The assigned address should already indicate in which lower layer DIF a node has registered. The address of the Global Network DIF would look as <region_id; unique_global_id > with region_id as the identifier of the current node's region and unique_global_id as an identifier that must be unique for the region. Similarly, in the Region and Area DIFs the addresses are $<$ area_id; unique_region_id $>$ and $<$ cell_id; unique_area_id $>$ respectively. Finally, in the Cell DIF, each vehicle should get an address in the form <unique_cell_id>. The address components unique_*_id should contain identifiers that are unique in the respective DIF, i.e. the Cell, Area, or Region.

Fig. 4 shows how the various DIF addresses change when a vehicle moves among cells, i.e. when it visits cells A, B, D, and F. Fig. 4 shows the four DIF addresses of the vehicle for the Global Network, Region, Area, and Cell DIF at each cell. The changed addresses during movement are underlined.

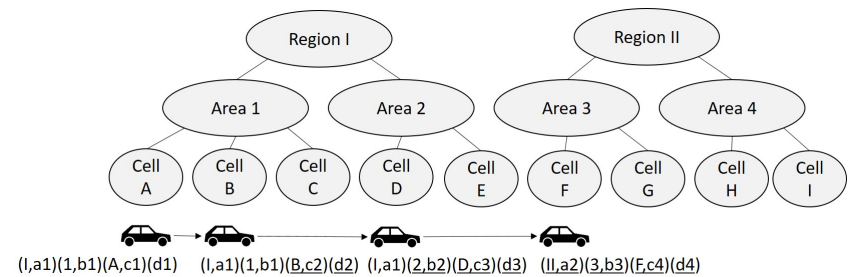

Fig. 4. Mobility Example

Topological addresses have the advantage that the routing decision can be derived from the address and that routing tables can be kept small due to possible aggregation of routing entries. Thus, topological addresses scale well in larger DIFs. Common address prefixes might indicate here that no intermediate node is needed, but the message can be sent directly to the destination process in the DIF. Topological addresses, however, have the disadvantage that even if a node stays within a Region or Area, the address might change if the topological address changes. For example, the address in Global Network DIF changes, if the node changes the underlying Region DIF. On a higher layer DIF the lower layer DIF must be known. For example, on the Region DIF, the area_id of a node must be known. This information is delivered to higher layers as a result of the registration process [3]. The analysis in Sec. IV shows that this overhead is limited and affordable.

To limit the load of address management, this task can be easily distributed among several management entities in a DIF. To each address assigning entity a block of addresses can be allocated, e.g., to all entities assigning an address in the Area DIF, a portion of the unique_area_id space can be allocated.

\section{Unicast Routing}

Routing in RINA DIF is performed as follows [3]: If source A wants to send a message to $\mathrm{D}$, it calls a function RRoute(A,D,m) to find the next-hop node (B in this case), and sends the message down to the lower-level DIF, which maps the source and next-hop addresses to their lower-level addresses and calls RRoute recursively, c.f., Fig. 5. A lower layer process $\mathrm{E}$ will forward the message to $\mathrm{F}$, which in turn will deliver it to process $\mathrm{B}$. Process $\mathrm{B}$ will repeat the same process to send the message to $\mathrm{C}$, which in turn sends the message to $D$. In this case, a message is routed over the path A - B - C - D in the higher layer DIF. Note that Fig. 5 does not show how processes $\mathrm{B}, \mathrm{C}$, and D are interconnected.

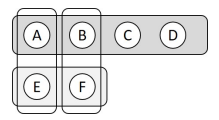

Fig. 5. Routing Example

If an application requests to send a unicast message to a particular destination application process (e.g., from the most left vehicle to the most right vehicle in Fig. 3), the request is delivered within the protocol stack of the left vehicle from the DAF to the Global Network DIF, after determining the next hop in the DAF. At the Global Network DIF, the destination application name is mapped to the global network address of the next hop in the DAF. In the Global Network DIF, the next hop in that DIF must be determined, i.e. it must be determined whether the message should be sent to a Backbone Router in that DIF or whether the message should be sent directly to the addressed Global Network DIF process. The message is then delivered to the Region DIF, with the destination address determined by the Global Network DIF, i.e., the next hop in the Region DIF. The next hop in the Region DIF is then either a vehicle or a Region Router. Similarly, in the Area DIF, the next hop in that DIF must be determined.

\section{E. Service Discovery and Anycasting}

Applications often rather care about accessing a service than a particular node offering such service. Service discovery can be supported by anycast addresses, where an anycast address is describes a particular service or a group of services. There are certain alternatives how to support service discovery.

- Available services can be registered in a (distributed) service directory, e.g., when applications providing a service have started up. A lookup for a service then results in the application name of the particular application providing that service. Service selection might depend on certain criteria such as performance metrics (e.g., network delays or number of hops) or current load of servers. This can only be supported if applications continuously update that information in the directory. Continuous updates might generate some load and could become inefficient in large networks.

- Another possibility is to deploy forwarding rules for service lookups or service requests inside the network. In contrast to a directory approach, binding of services to nodes is performed rather lately (late binding), which better allows to select services considering dynamic network and server characteristics. 
RINA supports both alternatives, i.e. service directories as well as late binding. A directory can be implemented as a separate service discovery DAF or as part of an applicationspecific DAF. In [2], so-called sentential addresses have been proposed. Such sentential addresses can be mapped network addresses, preferably in a DAF.

\section{F. Multi-homing}

RINA supports multi-homing as well. Fig. 6 shows the DIF architecture when a vehicle with two wireless interfaces joins two Cell DIFs simultaneously, possibly using two frequencies. Smooth handovers can be supported by simultaneously forwarding packets to both RSUs serving the vehicle.

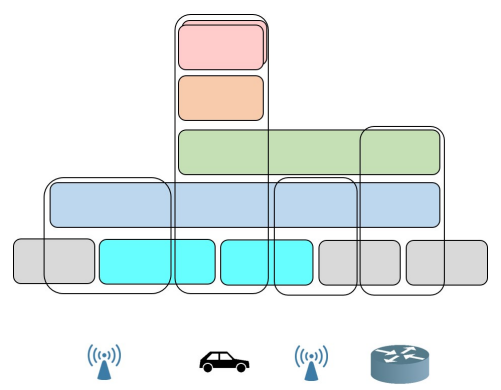

Fig. 6. Multi-Homing and DTNs in RINA

\section{G. Multiple Network Providers Supporting Heterogeneous Radio Technologies}

The described architecture in Sec. III-B only supports strictly geographically structured and hierarchical network topologies. However, there might be scenarios where this assumption does not hold. For example, it might happen that multiple network providers providing heterogeneous wireless access networks, e.g., 4G/LTE and WiFi, cover the same geographical area together. For such case, we propose to have an intermediate level of routers between the RSUs (or base stations, e.g., in case an area is covered by both RSUs and cellular network base stations) and the ARs. Such network allows to connect RSUs (or base stations) to interconnect with ARs and RRs.

\section{Performance Analysis}

This Section analyses the performance of V-RINA in terms of mobility management. We estimate the overhead that will be caused in the event that a vehicle changes its cell. In this case, a vehicle might register in new DIFs and deregister in other DIFs. Due to DIF changes the routing information must be updated. This is because adding and deleting nodes in DIFs, but also due to address changes in case of using the suggested topological addresses. In particular, when a vehicle joins an N-DIF, the topological address in the $(\mathrm{N}+1)$-DIF changes as well and routing information has to be updated. We assume that the effort to update routing information within a DIF consisting of $\mathrm{n}$ nodes has a complexity of $\mathrm{O}(\mathrm{n})$, since each node has to receive 1 message with the updated routing information. To calculate the probability that a vehicle has to change its area $(\mathrm{p}(\mathrm{AC}))$ in case of a cell change, we assume a grid like division of areas into cells, c.f. Fig. 7. If the node is within one of the interior (red) cells of an area, i.e. a cell with no neighbor cells in another area, the probability for an area change in case of a cell change is 0 . If the node is in one of the blue corner cells, the area change probability becomes $1 / 2$, since 2 of the 4 neighbor cells are within the own area, assuming that cell changes are only possible in north, south, east or west directions. The area change probability for the remaining (green) cells is 1/4. Assuming that there are $\mathrm{m}^{2}=\mathrm{M}$ cells per area $(\mathrm{m}>2)$, there are $4(\mathrm{~m}-1)-4=4 \mathrm{~m}-8$ remaining cells, 4 corner cells, and $\mathrm{m}^{2}-4(\mathrm{~m}-1)=(\mathrm{m}-2)^{2}$ inner cells.

$$
p(A C)=\frac{0 \cdot\left(m^{2}-4(m-1)\right)+\frac{1}{2} \cdot 4+\frac{1}{4} \cdot(4 m-8)}{m^{2}}=\frac{1}{m}
$$

With $m^{2}=$ M Eq. 1 becomes Eq. 2. Eq. 1 can be used to calculate the probability for a region change in case of a cell change. We simply have to replace $m^{2}$ by LM in Eq. 1 .

$$
\begin{aligned}
& p(A C)=\frac{1}{\sqrt{M}} \\
& p(R C)=\frac{1}{\sqrt{L M}}
\end{aligned}
$$

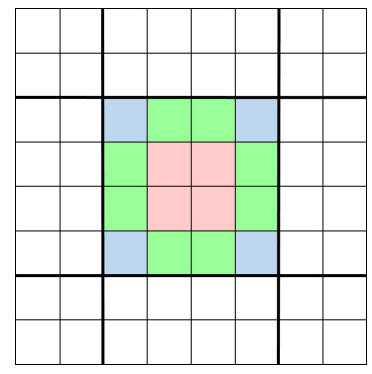

Fig. 7. Areas and Cells in a Grid

Next, we analyze the overhead induced by a cell, area, and region change. Algorithm 1 describes the messages to be exchanged in case of a cell change. We assume here that the number of vehicles per cell is equally distributed.

We distinguish three cases: a cell change only, a cell change plus an area change, and a cell change followed by both an area change and a region change.

1) Cell change: The number of messages to be exchanged for a cell change only (lines $1-6$ ) is given by

$$
2+\frac{2 \cdot N}{K \cdot L \cdot M}+1=3+\frac{2 \cdot N}{K \cdot L \cdot M}
$$

The main overhead is caused by updating the routing tables for the old Cell DIF and the new Cell DIF. A constant number of messages needs to be exchanged due to de-registering in the old Cell DIF and registering in the new Cell DIF. Moreover, the address in the Area DIF must be updated, since in the Area DIF we use topological addresses that depend on the current cell. 


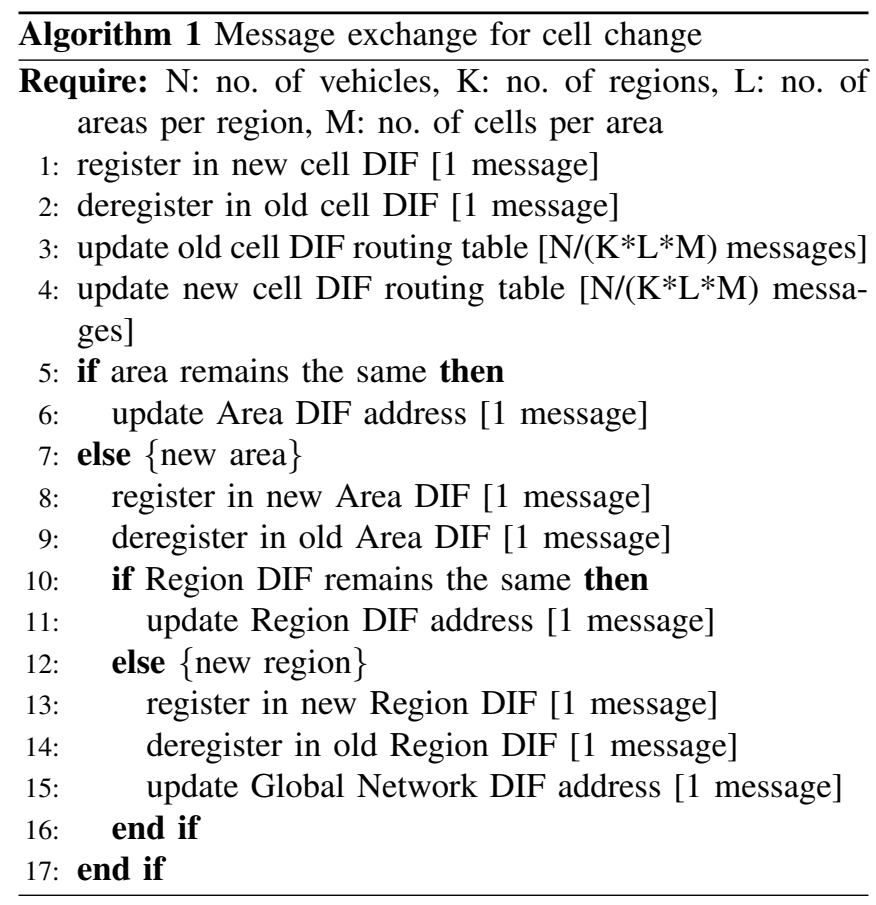

2) Area change: The benefits of using topological addresses become obvious when we analyse the messages needed on the Area DIF level. The use of topological addresses in the Area DIF allows us to build routing tables, which only depend on the subnets in the area. We assume that the topology in an area remains rather constant, as RSUs serving the cells are interconnected by fixed networks with infrequent changes. Thus, only two or three messages need to be exchanged for an area change, c.f. lines $7-11$.

3) Region change: If an area change also causes a region change, there are three additionally required messages (lines 12 - 15).

Eq. 1 - 4 can be combined to calculate the average number of messages to be exchanged in case of a cell change:

$$
3+\frac{2 N}{K L M}+\frac{3}{\sqrt{M}}+\frac{3}{\sqrt{L M}}
$$

In Eq. 5 the first summand is constant, the third and the fourth are $<3$ for both $\mathrm{L}$ and $\mathrm{M}>1$. The overhead is mainly determined by the second summand, which is the number of vehicles in a cell, and increases linearly with the average number of vehicles in a cell (N/KLM). Thus, we should limit the cell size by appropriately dividing regions into areas and areas into cells.

Another aspect is the signaling load to be handled by managers of Cells, Areas, and Regions. Vehicles can communicate at a WiFi hotspot for up to $1000 \mathrm{~m}$ [12]. Moreover, IEEE $802.11 \mathrm{p}$ is envisioned for ranges of up to $1000 \mathrm{~m}$. Assuming a speed of $100 \mathrm{~km} / \mathrm{h}$, communication is possible for 36s. Assuming some overlap of cells and that not more than 2 cell changes per minute can happen for a vehicle, the time between cell changes per vehicle is at least $30 \mathrm{~s}$.
The number of vehicles per hour passing certain points of roads in California is limited to approximately $10^{\prime} 000$ vehicles per hour ${ }^{1}$. Thus, not more than 10 '000 vehicles per hour are moving along a road from one cell to another. Assuming that a cell can be reached from four directions, not more than 40'000 vehicles per hour enter a cell. Thus, we could expect to have up to 40 '000 vehicles / $3600 \mathrm{~s}=$ 11.1 vehicles/s entering a cell. Assuming the number of cell changes per second is $\mathrm{Cc}$, the number of area changes is $\mathrm{Ac}$,

$$
A c=p(A C) \cdot C c
$$

Assuming $\mathrm{M}=100$ (in case of an area consisting of $10 \times 10$ cells), $\mathrm{Cc}=11.1$, the number of area changes per second is

$$
\begin{gathered}
A c=\frac{1}{10} \cdot 11.1=1.11 \\
\text { V. CONCLUSIONS }
\end{gathered}
$$

This paper discussed how to support vehicular networks by RINA. From the discussion, V-RINA seems to be well suited and scalable for such vehicular networks and supports various networking techniques, e.g. cell-based networks, and vehicular ad-hoc networks.

\section{ACKNOWLEDGMENTS}

This work has been supported by the Swiss National Science Foundation, project no. IZK0Z2_175514.

\section{REFERENCES}

[1] J. Day, I. Matta, and K. Mattar, "Networking is ipc: A guiding principle to a better internet," in Proceedings of the 2008 ACM CoNEXT Conference. New York, NY, USA: ACM, 2008, pp. 67:1-67:6.

[2] J. Day, Patterns in Network Architecture: A Return to Fundamentals. Prentice Hall, 2007.

[3] V. Ishakian, J. Akinwumi, F. Esposito, and I. Matta, "On supporting mobility and multihoming in recursive internet architectures," Comput. Commun., vol. 35, no. 13, pp. 1561-1573, Jul. 2012.

[4] G. Boddapati, J. Day, I. Matta, and L. Chitkushev, "Assessing the security of a clean-slate internet architecture," in 2012 20th IEEE International Conference on Network Protocols (ICNP), Oct 2012, pp. $1-6$.

[5] S. Wang, X. Zhang, Y. Zhang, L. Wang, J. Yang, and W. Wang, "A survey on mobile edge networks: Convergence of computing, caching and communications," IEEE Access, vol. 5, pp. 6757-6779, 2017.

[6] "Icarus: Improvement of traffic condition through an alerting and rerouting system," Computer Networks, vol. 110, pp. 118 - 132, 2016.

[7] F. A. Silva, A. Boukerche, T. R. M. B. Silva, L. B. Ruiz, E. Cerqueira, and A. A. F. Loureiro, "Vehicular networks: A new challenge for content-delivery-based applications," ACM Comput. Surv, vol. 49, pp. 11:1-11:29, 2016.

[8] S. Allal and S. Boudjit, "Geocast routing protocols for vanets: Survey and guidelines," in 2012 Sixth International Conference on Innovative Mobile and Internet Services in Ubiquitous Computing, July 2012, pp. 323-328.

[9] J. C. Navas and T. Imielinski, "Geocast - geographic addressing and routing," in Proceedings of the 3rd Annual ACM/IEEE International Conference on Mobile Computing and Networking, ser. MobiCom '97. New York, NY, USA: ACM, 1997, pp. 66-76.

[10] P. Li, T. Zhang, C. Huang, X. Chen, and B. Fu, "Rsu-assisted geocast in vehicular ad hoc networks," IEEE Wireless Communications, vol. 24, no. 1, pp. 53-59, February 2017.

[11] C. Castelluccia, "Hmipv6: A hierarchical mobile ipv6 proposal," SIGMOBILE Mob. Comput. Commun. Rev., vol. 4, no. 1, pp. 48-59, Jan. 2000.

[12] J. Ott and D. Kutscher, Exploiting Regular Hot-Spots for Drive-thru Internet. Berlin, Heidelberg: Springer Berlin Heidelberg, 2005, pp. 218-229.

\footnotetext{
${ }^{1}$ http://www.dot.ca.gov/trafficops/census/docs/2015_aadt_volumes.pdf
} 